\title{
PHASE TRANSITIONS IN PSEUDOSPIN-ELECTRON MODEL AT WEAK COUPLING
}

\author{
I. V. Stasyuk, T. S. Mysakovych \\ Institute for Condensed Matter Physics of the National Academy of Science of Ukraine, \\ 1 Svientsitskii St., UA-79011 Lviv, Ukraine
}

(Received October 2, 2001)

\begin{abstract}
The thermodynamically equilibrium states and phase transitions in the pseudospin-electron model with the transverse field are studied in the weak coupling approximation. In the regime of the constant chemical potential the possibility of the first-order phase transitions between uniform phases as well as the first- or the second-order transitions into phase with the doubled lattice period is established depending on the value of $\mu$. The conditions of appearance of the phase separation in the system at the given average electron concentration are analysed. The influence of the transverse field (which is connected with the tunneling-like splitting) on the phase transitions and the conditions of their realization is also studied in the paper. The phase diagrams are built at the various electron densities of states in the conduction band.
\end{abstract}

Key words: pseudospin-electron model, Falicov-Kimball model, phase transition, weak coupling, phase separation, chess-board phase.

PACS number(s): 63.20.Ry, 64.70.Kb, 71.10.Fd, 77.80.Bh

\section{INTRODUCTION}

The pseudospin-electron model (PEM) is one of the models which were formulated in recent times and are intensively investigated in the theory of the strongly correlated electron systems. This model appeared in connection with the investigation of the role of the locally anharmonic structure elements in the high temperature superconductors (HTSC) in the formation of their electron spectrum and in the pairing correlations which lead to the appearance of the superconducting state. Anharmonicity of the vibrations of ions from certain sublattices is described in the model with the help of pseudospin formalism; in the case $S=\frac{1}{2}$ two lowest vibrational states in the anharmonic potential well are taken into account only.

At the standard formulation of the PEM the electron subsystem is described by the Hubbard Hamiltonian whereas the interaction of electrons with anharmonic subsystem is caused by the influence of the internal field depending on the pseudospin orientations. The model Hamiltonian includes the electron transfer $(t-$ term), electron correlation( $U$-term), pseudospin-electron interaction ( $g$-term); energy of the tunneling-like splitting of local levels ( $\Omega$-term) and asymmetry of the local anharmonic potential ( $h$-term) are also taken into account [1-4]:

$$
\begin{aligned}
H & =\sum_{i}\left(U n_{i, \uparrow} n_{i, \downarrow}-\mu\left(n_{i, \uparrow}+n_{i, \downarrow}\right)+g S_{i}^{z}\left(n_{i, \uparrow}+n_{i, \downarrow}\right)\right. \\
& \left.-h S_{i}^{z}-\Omega S_{i}^{x}\right)+\sum_{i, i} t_{i j} c_{i}^{\dagger} c_{j}
\end{aligned}
$$

The investigations of this model in recent years were concerned with the analysis of the structure of the electron spectrum with an allowance for the strong short range correlations [5], to the investigation of the charge and pseudospin correlations and dielectric susceptibility $[6,7]$ as well as pair correlations of the supercunducting type [3]. Different aspects of the PEM thermodynamics (in particular, equilibrium and metastable states and bistability phenomena [8], phase transitions between states with different pseudospin orientation and electron concentration in the regime of the fixed electron chemical potential [9], transitions into the phase separated states at the given value of the average electron concentration [10]) were the subject of attention too. Actuality of such investigations for HTSC objects is connected in particular with the problem of genesis of the crystal structure inhomogeneities (see, for example [11]) or the so called stripe structures [12] in these systems.

The simplified PEM (at $\Omega=0$ and $t=0$, but with the inclusion of the direct pseudospin-pseudospin interaction) was the subject of consideration in [9]. The possibility of the first order phase transitions between uniform states with the jumps of the pseudospin mean value as well as of the phase separation were revealed. Similar results were obtained for the case $t \neq 0, \Omega=0$ at the large values of the interaction constant $g$ both in the limit $U \rightarrow \infty[13]$ and for the simplified variant of the model at $U=0$ [14]; the last consideration was carried out in the framework of the thermodynamically consistent scheme of the generalized random phase approximation (GRPA) [15]. The possibility of the appearance of the modulated (the so-called chess-board) phase was shown for the simplified PEM $(U=0, \Omega=0)$ in the case of strong coupling $(g \gg W, W$ is a half width of the electron energy band). Phase diagrams which describe transitions between homogeneous and modulated phases at the change of the field $h$, the electron chemical potential $\mu$ and the temperature $T$ were built.

It should be noted that the PEM has certain similarity to the known in literature and intensively investigated in recent times Falicov-Kimball (FK) model [16], in which 
the interaction of localized and moving electrons is taken into account. The mentioned model is a simplified variant of the Hubbard model and is used at the investigations of the mentioned above spatial inhomogeneities in the systems with the strongly correlated electrons. The variety of homogeneous and modulated phases, transitions between them, the possibility of the phase separations is characteristic of the Falicov-Kimball model [17-21]. In comparison with the PEM there exists, however, a difference in the regime of thermodynamical averaging. Localized and moving particles in the electron FK model possess the common chemical potential; this is absent in the PEM. Besides, the term with transverse field $S_{i}^{x}$ which is responsible for the own dynamics in localized particle subsystem is absent in the FK model. It is evident that thermodynamics of the PEM can have its own special features and can include the effects which are not presented in the FK model.

There is also an analogy between the simplified PEM $(\Omega=0)$ and the electron Hamiltonian of the annealed binary alloy model (see, for example [18]), but in this case the models differ in thermodynamical regime (in the PEM the field acting on pseudospins is fixed, while in the alloy model the component concentrations are given which corresponds to the fixed value of $\left.\left\langle S^{z}\right\rangle\right)$.

The aim of this work is the consideration of thermodynamics of the simplified $(U=0)$ PEM in the case of weak coupling, when the interaction constant $g$ is small in comparison with the width of the electron band $2 W$. This case has not come under attention by now. Work [13] can be mentioned only; it was shown there at the consideration of the PEM with $U=0, \Omega=0$ in the framework of the dynamic mean field method that the electron band does not split when $g \lesssim W$. Most of the previous works on the PEM thermodynamics were carried out for the large $g$ case basing on the approaches in which the splitting in electron spectrum is taken into account. The consideration was restricted to the case of $\Omega=0$; the presence of the transverse field $\Omega S_{i}^{z}$ leads to certain difficulties in the GRPA scheme. In contrast to this we do not restrict ourselves in the present investigation to the case $\Omega=0$ only. The changes in the band spectrum at the weak coupling with pseudospins and presence of the tunneling (or pseudo-tunneling) splitting can have significant influence on the phase transition picture, modifying thermodynamical criteria of the appearance of modulated phases. In this work the calculation of the thermodynamical functions of the PEM with $U=0$ and the investigation of the thermodynamically stable states and phase transitions within the uniform phases and into the modulated phase (with the doubling of a lattice period) are carried out.

\section{THERMODYNAMICS}

The investigation of the PEM in the weak coupling approximation will be carried out here analogously to the traditional consideration of the weak one-site correlation $U$. In the case $U \ll W$ the Hartree-Fock ap- proximation is applied, using the decoupling $U n_{\sigma} n_{-\sigma} \rightarrow$ $U\left[n_{\sigma}\left\langle n_{-\sigma}\right\rangle+n_{-\sigma}\left\langle n_{\sigma}\right\rangle-\left\langle n_{\sigma}\right\rangle\left\langle n_{-\sigma}\right\rangle\right]$. It gives the possibility to describe satisfactorily magnetic properties of the Hubbard model in the case when the correlational splitting of the electron band is absent (see, for example $[22,23]$ ) (such splitting takes place when $W<U$ and can be described in the simplest way by using the HubbardI approximation). At the intermediate correlation energies $(U \sim W)$ the metal-insulator transition, at which the gap in the spectrum dissapears at the decrease of $U$, takes place in the Hubbard model.

For the simplified PEM with $U=0$ the interaction constant $g$ plays the role which is similar to that of the energy $U$ in the Hubbard model. At the decrease of the constant $g$ below the critical value $(g \sim W)$ the system should pass to the mean field regime of the Hartree-Fock type.

Basing on the above mentioned arguments we use the approximation

$$
g n_{i} S_{i}^{z} \rightarrow g n_{i}\left\langle S_{i}^{z}\right\rangle+g\left\langle n_{i}\right\rangle S_{i}^{z}-g\left\langle n_{i}\right\rangle\left\langle S_{i}^{z}\right\rangle,
$$

where the pseudospin-electron interaction is taken into account in the spirit of the mean field approximation (MFA) through an internal self-consistent field which acts on electrons and pseudospins.

In the following we will consider two different cases. The first one is the case of the uniform field, which is the same in all lattice sites. This corresponds to the homogeneous pseudospin ordering and the spatially uniform (independent on the site number) electron concentration. The second one is the case of modulation with doubling of the lattice period, with which the corresponding changes of the mean values $\left\langle S_{i}^{z}\right\rangle$ and $\left\langle n_{i}\right\rangle$ are connected.

\section{A. Uniform phase}

Let us consider the uniform case: $\left\langle\sum_{\sigma} n_{i \sigma}\right\rangle=n$, $\left\langle S_{i}^{z}\right\rangle=\eta$. The Hamiltonian of the PEM in the MFA is as follows:

$$
\begin{aligned}
& H=H_{e l}+H_{s}+U, \\
& H_{e l}=\sum_{i, \sigma}(g \eta-\mu) n_{i, \sigma}+\sum_{i, j, \sigma} t_{i, j} c_{i, \sigma}^{\dagger} c_{j, \sigma}, \\
& H_{s}=\sum_{i}\left[(g n-h) S_{i}^{z}+\Omega S_{i}^{x}\right], \\
& U=-g \sum_{i} n \eta=-N g n \eta,
\end{aligned}
$$

here $N$ is the number of the lattice sites. Passing to $k$ representation and performing the transformation

$$
\begin{aligned}
& S_{i}^{z}=\sigma_{i}^{z} \cos \theta+\sigma_{i}^{x} \sin \theta \\
& S_{i}^{x}=\sigma_{i}^{x} \cos \theta-\sigma_{i}^{z} \sin \theta
\end{aligned}
$$




$$
\begin{aligned}
\sin \theta & =\frac{\Omega}{\lambda}, \cos \theta=\frac{h-g n}{\lambda}, \\
\lambda & =\sqrt{(g n-h)^{2}+\Omega^{2}}
\end{aligned}
$$

we will diagonalize $H_{e l}, H_{s}$ :

$$
\begin{aligned}
& H_{e l}=\sum_{k, \sigma}\left(g \eta-\mu+t_{k}\right) n_{k \sigma}, \\
& H_{s}=-\lambda \sum_{i} \sigma_{i}^{z},
\end{aligned}
$$

here $t_{k}=\sum_{i-j} t_{i j} e^{i \mathbf{k}\left(\mathbf{R}_{i}-\mathbf{R}_{j}\right)}$. The electron band $E_{k}=$ $g \eta+t_{k}$ in this case changes its position depending on the average value of pseudospin $\eta$. Its displacement with respect to the chemical potential level leads to the change of the electron states occupancy and results in the change of the electron concentration. This in its turn influences the value of the field acting on pseudospins and determining their mean value. This self-consistent connection is described by the following set of equations for the parameter $\eta$ and electron concentration $n$ :

$$
\begin{aligned}
& n=\frac{1}{N} \sum_{k \sigma}\left(e^{\beta\left(g \eta+t_{k}-\mu\right)}+1\right)^{-1} \equiv \frac{1}{N} \sum_{k \sigma} f\left(E_{k}-\mu\right), \\
& \eta=\frac{h-g n}{2 \lambda} \tanh \left(\frac{\beta \lambda}{2}\right), \quad \beta=\frac{1}{T} .
\end{aligned}
$$

The grand canonical potential (per one lattice site) in the MFA is given by the expression

$$
\begin{aligned}
\frac{\Phi}{N} & =-\frac{T}{N} \sum_{k, \sigma} \ln \left(1+e^{\left(\mu-t_{k}-g n\right) / T}\right) \\
& -T \ln \left(2 \cosh \frac{\beta \lambda}{2}\right)-g n \eta .
\end{aligned}
$$

From the point of view of thermodynamical equilibrium we can distinguish two regimes: (i) the constant electron chemical potential; (ii) the given electron concentration $[6,9]$. Stable equilibrium states in the regime $\mu=$ const can be found from the condition of minimum of the grand canonical potential $\Phi$ and in the regime $n=$ const they can be found from the condition of minimum of the free energy $F=\mu n+\Phi$.

$$
\text { 1. Regime } \mu=\mathrm{const}
$$

It should be noted that the set of equations ( 7 ) is invariant with respect to the transformation

$$
\begin{aligned}
& \mu \rightarrow-\mu ; h \rightarrow 2 g-h, \\
& n \rightarrow 2-n ; \eta \rightarrow-\eta
\end{aligned}
$$

In the symmetric case $\mu=0, h=g$ system (7) can be transformed to the form:

$$
\begin{aligned}
& \xi=\frac{1}{N} \sum_{k} \operatorname{th} \frac{\beta}{2}\left(t_{k}+g \eta\right), \\
& \eta=\frac{g}{2 \lambda} \xi \tanh \frac{\beta \lambda}{2}
\end{aligned}
$$

where $\xi=1-n$. The solution

$$
\eta=0, n=1(\xi=0)
$$

exists at any temperatures and describes disordered phase. Besides this solution there appears at low temperatures the non-zero solution $\eta \neq 0, \xi \neq 0$. A critical temperature $T_{c}$, which in this case corresponds to the second order phase transition point, is determined from the equation:

$$
1+\frac{g^{2}}{2 \Omega} \tanh \frac{\beta \Omega}{2} \Pi_{0}=0 .
$$

Equation (12), which is obtained by linearization of the set of equations (7), in the case $\Omega \rightarrow 0$ reduces to the form:

$$
1+\frac{\beta}{4} g^{2} \Pi_{0}=0
$$

Here

$$
\Pi_{0}=\frac{2}{N} \sum_{k} f^{\prime}\left(t_{k}\right)=2 \int_{-W}^{W} d t \rho(t) f^{\prime}(t)
$$

where $\rho(t)=\frac{1}{N} \sum_{k} \delta\left(t-t_{k}\right)$ is the density of electron states (DOS), connected with the electron transfer; the function $t_{k}$ changes in the interval $[-W, W]$. In the low temperatures limit

$$
\Pi_{0}=-\left.2 \rho(\mu)\right|_{\mu=0}, \quad T_{c}=\frac{g^{2}}{2} \rho(0),
$$

when the DOS at the Fermi level $\rho(\mu)$ is finite (that takes place for the three-dimensional lattice or for the twodimensional lattice when the rectangular DOS $\rho(t)=$ $\frac{1}{2 W}$ is used approximately). The real DOS for the twodimensional lattice possesses the Van-Hove singularity (see, for example [24])

$$
\left.\rho(t)\right|_{|t| \ll W} \approx \frac{2}{W \pi^{2}} \ln \frac{4 W}{|t|}
$$

which leads instead of (15) to the expression

$$
\Pi_{0} \cong-\frac{4}{W \pi^{2}} \ln \frac{4 W e}{2 T}
$$


and respectively to the equation for $T_{c}$ :

$$
1=\frac{2 g^{2}}{\pi^{2} \Omega W} \tanh \frac{\beta \Omega}{2} \ln \frac{4 W e}{2 T} .
$$

The solutions of equation (12) as functions of the interaction constant at different values of the transversal field parameter $\Omega$ are presented in Fig. 1 (we consider two cases: (i) the case when the direct momentum summation is used to calculate $\Pi_{0}$ (it corresponds to the DOS with the logarithmic singularity (16)) (ii) the case when we use the rectangular state density). In our calculations (here and in the following) we put $W=1$.

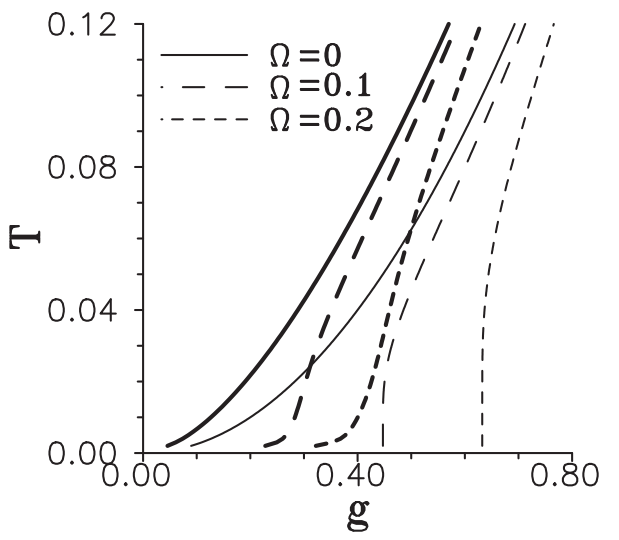

Fig. 1. The dependence of the critical temperature on the interaction constant $g$. Thick (thin) lines refer to the case when the direct momentum summation (rectangular DOS) is used when calculating $\Pi_{0}$, respectively.

It is worth mentioning that at the absence of singularity of the DOS there exists such a critical value

$$
\Omega_{\mathrm{cr}}=g^{2} \rho(0)
$$

$\left(\Omega_{\mathrm{cr}}=\frac{g^{2}}{2 W}\right.$ for the rectangular DOS), above which (at $\Omega>\Omega_{\text {cr }}$ ) the phase transition to ordered phase disappears. This is equivalent to the existence of a critical value of $g$ : at given $\Omega$ the phase transition is possible when $g>g_{c r}=\sqrt{\frac{\Omega}{\rho(0)}}$. In the case of the DOS with the logarithmic singularity the critical temperature exists at any $\Omega$ values and at $\frac{\Omega}{g} \gg \frac{g}{W}$ we have an asymptotic expresssion:

$$
T_{c} \approx \frac{4 e W}{2} \exp \left(\frac{-\Omega W \pi^{2}}{2 g^{2}}\right)
$$

The physical nature of the considered here phase transition at $h=g, \mu=0$ (the fixed $\mu$ regime) is the following: the appearance of the ordered phase is connected with its stabilization due to the shift of the electron band down to the low energy values under the influence of the internal field; this ensures the corresponding gain in the electron energy.

This mechanism remains the main reason of the phase transition when the initial electron band is not half-filled. In such a case (when $\mu \neq 0$ ) we performed the investigation using the numerical calculations when the set of equations ( 7 ) is solved and using the expression (8) for the grand canonical potential $\Phi$. The selection of the solutions was carried out using the condition of the absolute minimum of $\Phi$.
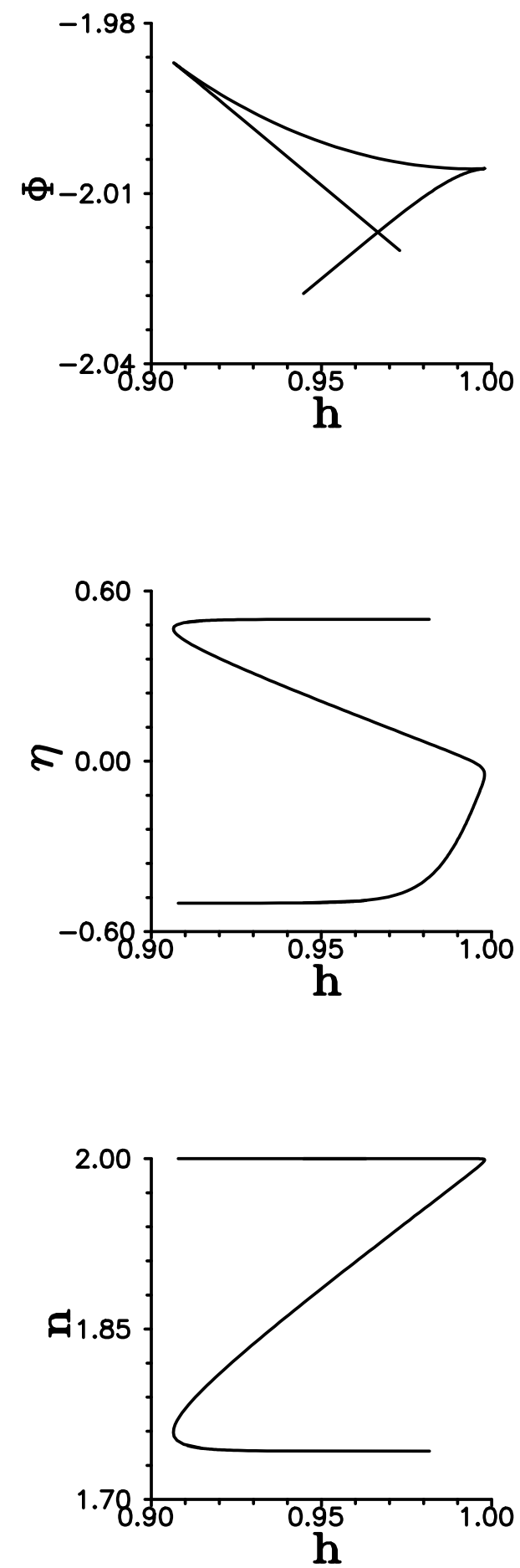

Fig. 2. The field dependence of the electron concentration, pseudospin mean value and grand canonical potential; $T=0.008$. 
The typical dependencies $\eta(h), n(h), \Phi(h)$ at certain temperature are shown in Fig. 2 (the case $\mu=W, \Omega=0$ is presented, rectangular density of states was used).

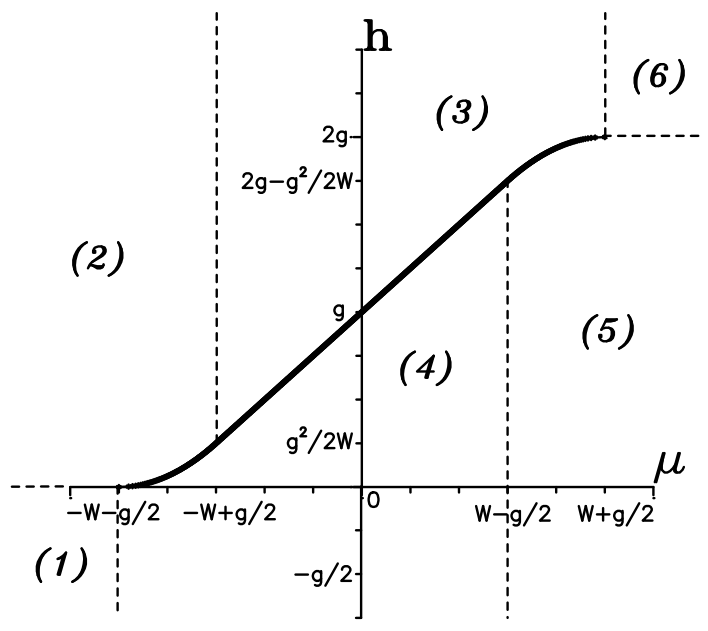

Fig. 3. The ground state diagram $(T=0, \Omega=0)$. Regions with the different $n, \eta$ values are separated by the dashed lines and the solid line (the phase transition line): (1) $n=0$, $\eta=1 / 2$; (2) $n=0, \eta=1 / 2$; (3) $\eta=1 / 2, n=1+\frac{\mu}{W}-\frac{g}{2 W}$; (4) $\eta=-1 / 2, n=1+\frac{\mu}{W}+\frac{g}{2 W}$; (5) $n=2, \eta=-1 / 2$; (6) $n=2, \eta=1 / 2$.

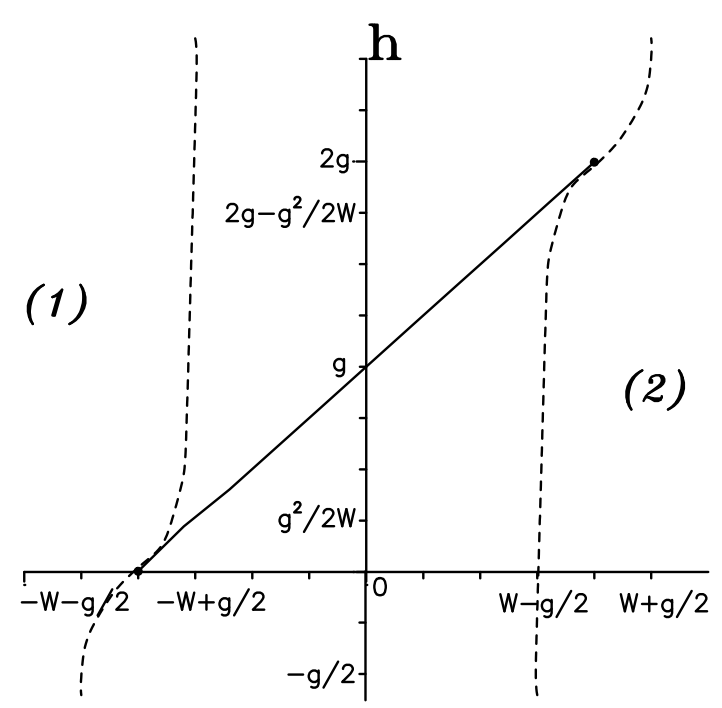

Fig. 4. The $(h, \mu)$ phase diagram $(T=0.004, \Omega=0.12)$. Regions with different $n, \eta$ values are separated by dashed lines and a solid line (the phase transition line):
(1) $n \approx 0, \eta \approx \frac{1}{2} \frac{h}{\sqrt{h^{2}+\Omega^{2}}}$
(2) $n \approx 2, \eta \approx \frac{1}{2} \frac{h-2 g}{\sqrt{(h-2 g)^{2}+\Omega^{2}}}$.

The system undergoes the first order phase transition with jumps of the mean values of the electron concentration and pseudospin at the change of the field $h$, the phase transition point is determined using the Maxwell rule. The similar transition takes place at the change of the chemical potential at fixed $h$. The dependences $n(h), \eta(h)$ in the case $\Omega \neq 0$ are similar to that in the case $\Omega=0$, the presence of the tunneling-like splitting decreases the temperature of the phase transition at the fixed values of $\mu$ and $h$.
The regions of coexistence of phases with different values of the electron concentration and pseudospin are shown in the plane $(\mu, h)$ at $\Omega=0$ and $\Omega \neq 0$ in Figs. 3 and 4 .

In the case $\Omega=0, T=0$ the phase transition line can be found in the analytic form:

$$
\begin{aligned}
& -W-\frac{g}{2}<\mu<-W+\frac{g}{2}: h=\frac{g^{2}}{2 W}\left(0.5+\frac{W}{g}+\frac{\mu}{g}\right)^{2} \\
& -W+\frac{g}{2}<\mu<W-\frac{g}{2}: h=g+\frac{g}{W} \mu \\
& W-\frac{g}{2}<\mu<W+\frac{g}{2}: h=2 g-\frac{g^{2}}{2 W}\left(0.5+\frac{W}{g}-\frac{\mu}{g}\right)^{2} .
\end{aligned}
$$

In the case $\Omega \neq 0$ numerical calculation is applied to determine the phase transition line; in the regions (1), (2) the electron concentration $n$ is near (but not exactly equal) to 0 or 2 respectively at low temperatures; at $T=0$ the phase transition takes place for the following values of the chemical potential:

$-W-\frac{g}{2} \sqrt{1-\left(\frac{2 \Omega W}{g^{2}}\right)^{\frac{2}{3}}}<\mu<W+\frac{g}{2} \sqrt{1-\left(\frac{2 \Omega W}{g^{2}}\right)^{\frac{2}{3}}}$.

Phase transition lines in the plane $(T, h)$ at different values of $\mu$ are shown in Fig. 5. Such line is vertical for the case $\mu=0$ only; for the case $\mu \neq 0$ the line is bent. This gives a possibility of the first order phase transition at the change of temperature (with the jumps of the parameters $\eta, n)$. The slopes of the phase equilibrium curves are opposite for $\mu>0$ and $\mu<0$. The lines of the critical points, in which the corresponding equilibrium curves at different $\mu$ end, are shown for the cases $\Omega=0, \Omega \neq 0$; the calculations are carried out using rectangular density of states.

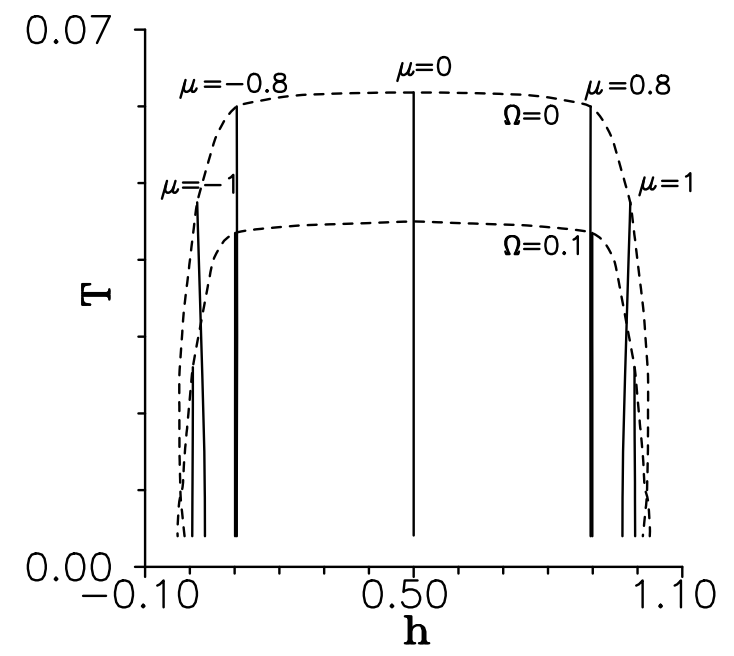

Fig. 5. The critical temperature lines (dashed lines) and the phase transition lines (solid lines) for $\Omega=0$ and $\Omega=0.1$ (the case of the rectangular DOS). 
Similar phase diagrams were obtained using direct momentum summation for square lattice (Fig. 6). As in the previous case the maximum critical temperature is achieved at $\mu=0$.
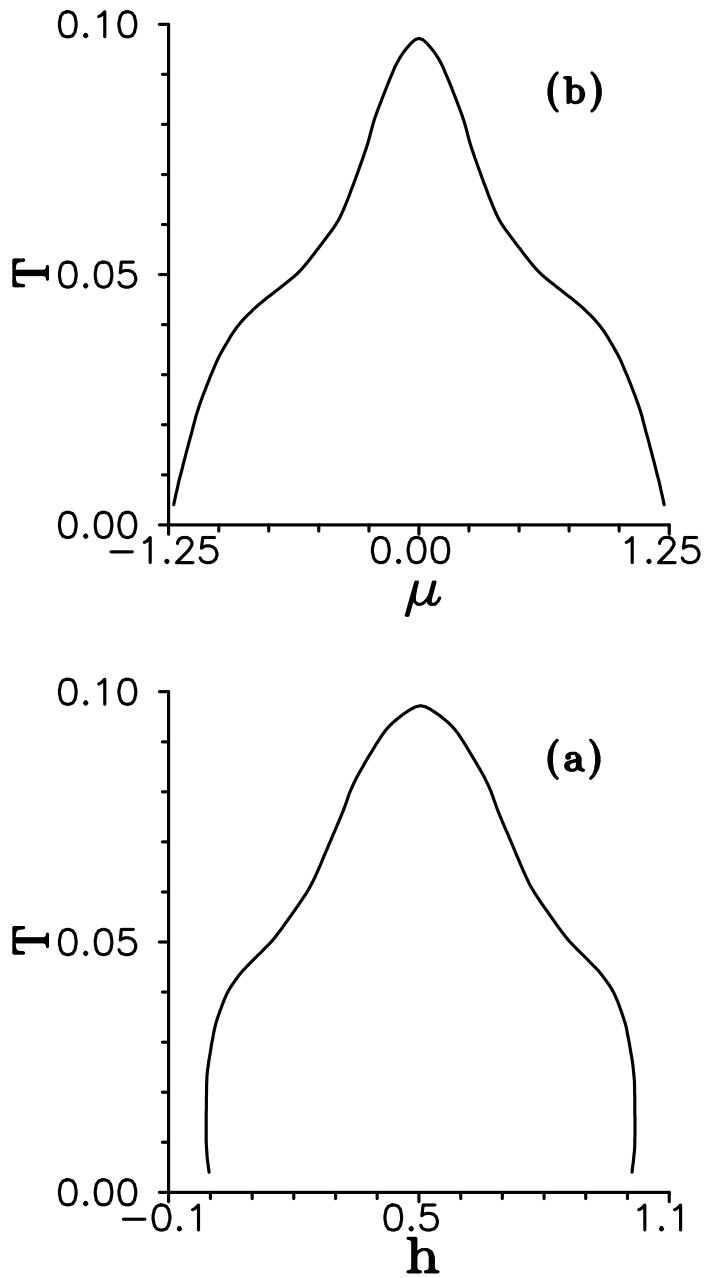

Fig. 6. The critical temperature lines on the a) $(T, h)$, b) $(T, \mu)$ planes for $\Omega=0$ (direct momentum summation is used).

\section{Regime $n=\mathrm{const}$}

The values $n_{1}, n_{2}, \eta_{1}, \eta_{2}$, between which the jumps of the electron concentration and pseudospin mean value take place at the phase transitions in the $\mu=$ const regime, correspond to the phases which coexist in the phase transition points. In the regime $n=$ const the phase separation on the phases with the above mentioned values of $n, \eta$ takes place. The instability with respect to phase separation is confirmed by the descending behaviour of the chemical potential as a function of $n$ in the corresponding region of parameter values. It is shown in Fig. 7, where the dependence of the band edges on the electron concentration is depicted as well.

At the used parameter values $(\Omega=0, h=0.7, T=$ $0.008)$ the system is unstable with respect to the phase separation in the region $n_{1}=1.149<n<n_{2}=1.645$. The values $n_{1}$ and $n_{2}$ correspond to the Maxwell rule.

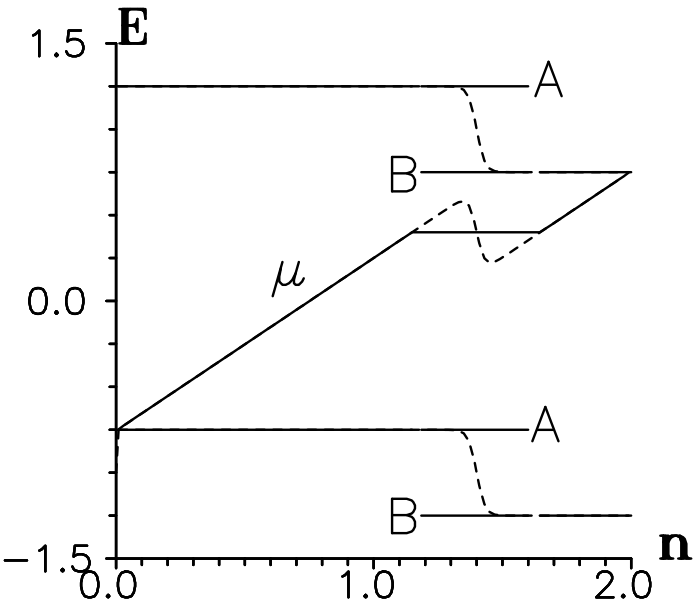

Fig. 7. The dependence of the band edges and the chemical potential on the electron concentration. $T=0.008, h=0.7$, $\Omega=0$. ( $A$ and $B$ denote the electron band edges for phases which coexist in the phase separation region).
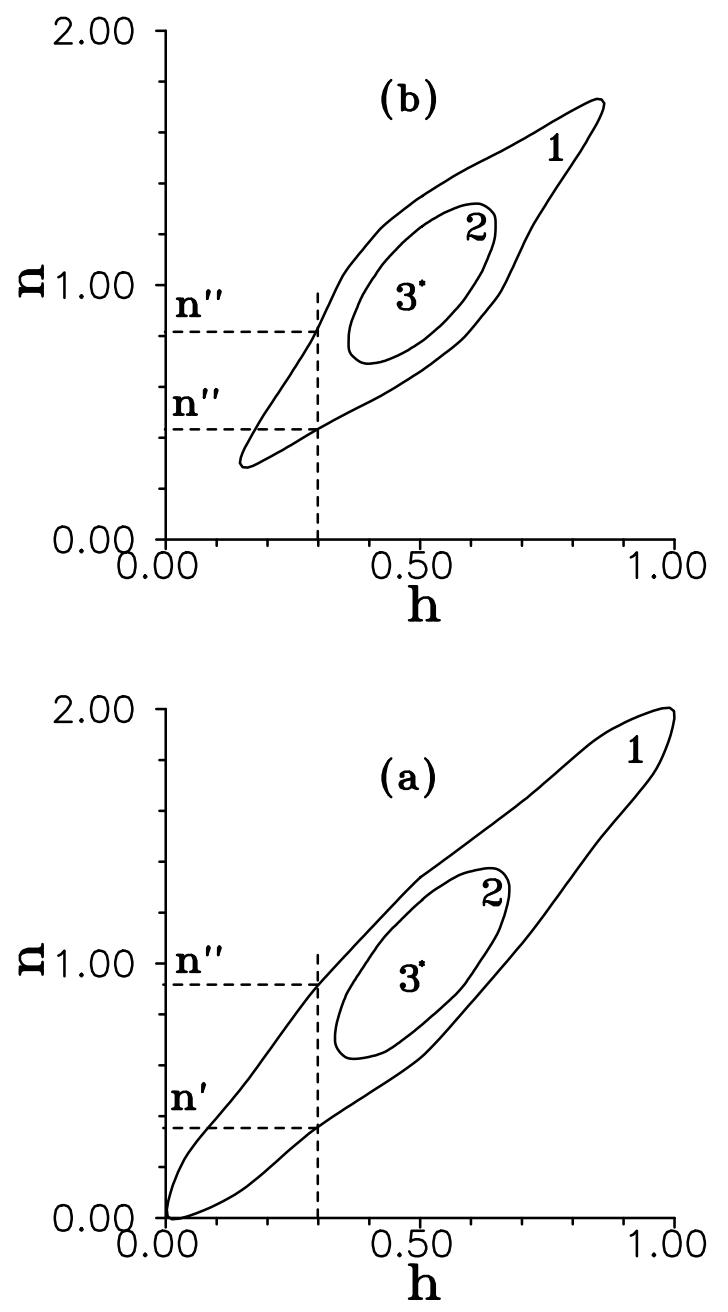

Fig. 8. $(n, h)$-phase diagram in the cases a) $\Omega=0$; b) $\Omega=0.1$. Phase separation regions for different temperatures are shown: a) $1-T=0.008,2-T=0.08,3$ $-T=0.0972$; b) $1-T=0.008,2-T=0.075,3-$ $T=0.0903$. 


\section{V. STASYUK, T. S. MYSAKOVYCH}

Phase separation regions are shown in Fig. 8 for different temperatures. The calculations were carried out for square lattice using direct momentum summation when solving the set of equations (7). At the increase of temperature the separation region narrows and at $T>T_{c}$ dissapears. This means that the system becomes homogeneous. The presence of the tunneling-like splitting leads to the decrease of the area of phase separation region and to the lowering of $T_{c}$.

The analytic expression for the border lines of the phase separation region can be obtained in the case $T=0, \Omega=0$ and the rectangular density of states:

$$
\begin{aligned}
& 0<h<\frac{g^{2}}{2 W}: n^{\prime}=0 ; \quad n^{\prime \prime}=\sqrt{\frac{2}{W} h}, \\
& \frac{g^{2}}{2 W}<h<2 g-\frac{g^{2}}{2 W}: n^{\prime}=\frac{h}{g}-\frac{g}{2 W} ; \quad n^{\prime \prime}=\frac{h}{g}+\frac{g}{2 W}, \\
& 2 g-\frac{g^{2}}{2 W}<h<2 g: n^{\prime}=2-\sqrt{\frac{2}{W}(2 g-h)} ; \quad n^{\prime \prime}=2 .
\end{aligned}
$$

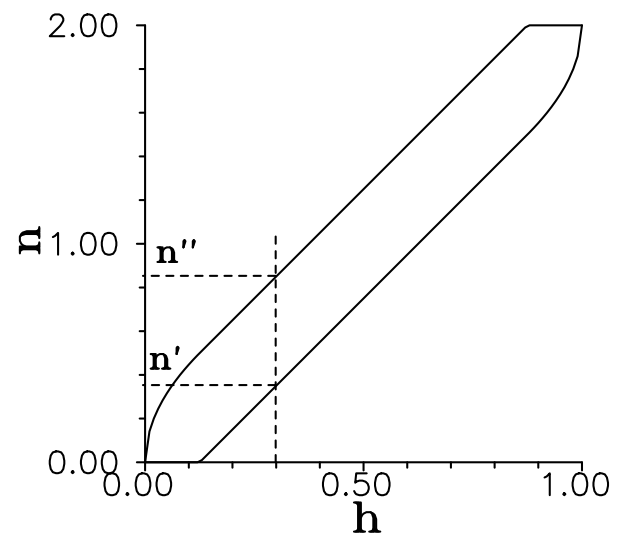

Fig. 9. $(n, h)$-phase diagram in the case $\Omega=0, T=0$; the rectangular DOS is used.

The corresponding $(n, h)$ phase separation diagram is shown in Fig. 9.

It can be mentioned that at weak coupling we have one phase separation region in the $(n, h)$ plane while in the strong coupling case $(g \gg W)$ there exist two such regions at the distance of order $g$ along $h$ axis; the modulated phase is intermediate between them [14].

\section{B. The case of the phase with double modulation}

In the framework of the carried out above investigation the phase transitions between phases with the uniform ordering (which are characterized by the different values of $n, \eta$ ) in the case of weak coupling were considered. As in the case $g \gg W$ the effective interaction between pseudospins (or electrons) which is formed by the direct pseudospin-electron coupling lies at the basis of such transitions. This interaction, as it is shown in [14], can result in the appearance of the modulated phase with doubling of the initial lattice period at the large values of the constant $g$ (when the chemical potential is located between the split due to the interaction subbands). Let us consider such possibility for the case of weak coupling $(g<W)$, which is the subject of our investigation.

At the double modulation of the lattice period the crystal is divided into two sublattices. We introduce the notations: $\eta_{\alpha}=\left\langle S_{i \alpha}^{z}\right\rangle, n_{\alpha}=\sum_{\sigma}\left\langle n_{i \alpha \sigma}\right\rangle(\alpha=1,2$ is the sublattice index), $i$ is an elementary cell index. Similarly to the case of the homogeneous phase we use the mean field approximation and write the Hamiltonian in the form

$$
\begin{aligned}
& H=H_{\mathrm{el}}+H_{s}+U \\
& H_{\mathrm{el}}=\sum_{i \alpha \sigma}\left(g \eta_{\alpha}-\mu\right) n_{i \alpha \sigma}+\sum_{i \alpha, j \beta} t_{i j}^{\alpha \beta} c_{i \alpha \sigma}^{\dagger} c_{j \beta \sigma} \\
& H_{\mathrm{s}}=\sum_{i, \alpha}\left[\left(g n_{\alpha}-h\right) S_{i \alpha}^{z}+\Omega S_{i \alpha}^{x}\right] \\
& U=-g \sum_{i \alpha} n_{\alpha} \eta_{\alpha} .
\end{aligned}
$$

Passing to $k$-representation and doing an unitary transformation

$$
\begin{aligned}
& c_{k_{1} \sigma}=\cos \phi \tilde{c}_{k_{1} \sigma}+\sin \phi \tilde{c}_{k_{2} \sigma}, \\
& c_{k_{2} \sigma}=-\sin \phi \tilde{c}_{k_{1} \sigma}+\cos \phi \tilde{c}_{k_{2} \sigma},
\end{aligned}
$$$$
\sin 2 \phi=\frac{t_{k}}{\sqrt{\left(g \frac{\eta_{1}-\eta_{2}}{2}\right)^{2}+t_{k}^{2}}}, \cos 2 \phi=\frac{-g \frac{\eta_{1}-\eta_{2}}{2}}{\sqrt{\left(g \frac{\eta_{1}-\eta_{2}}{2}\right)^{2}+t_{k}^{2}}},
$$$$
\left.t_{k}^{11}=t_{k}^{22}=0, t_{k}=t_{k}^{12}=t_{k}^{21}=\sum_{i-j} t_{i j}^{12} e^{i \mathbf{k}\left(\mathbf{R}_{i 1}-\mathbf{R} j 2\right.}\right)
$$

we will diagonalize $H_{\mathrm{el}}$ :

$$
\begin{aligned}
& H_{\mathrm{el}}=\sum_{\alpha \sigma k}\left(\lambda_{k \alpha}-\mu\right) \tilde{n}_{k \alpha \sigma}, \\
& \lambda_{k \alpha}=g \frac{\eta_{1}+\eta_{2}}{2}+(-1)^{\alpha} \sqrt{\left(g \frac{\eta_{1}-\eta_{2}}{2}\right)^{2}+t_{k}^{2}} .
\end{aligned}
$$

Similarly to $(5)$ we will diagonalize $H_{s}$ :

$$
H_{\mathrm{s}}=-\sum_{i \alpha} \tilde{\lambda}_{\alpha} \sigma_{i \alpha}^{z}, \quad \tilde{\lambda}_{\alpha}=\sqrt{\left(g n_{\alpha}-h^{2}\right)+\Omega^{2}} .
$$

Double modulation leads to the splitting in the electron spectrum due to the difference between the internal 
fields, acting in sublattices (the similar effect takes place in the FK model [19]). The initial band is divided into two subbands separated by the gap $\Delta=g\left|\eta_{1}-\eta_{2}\right|$ (see Fig. 10).

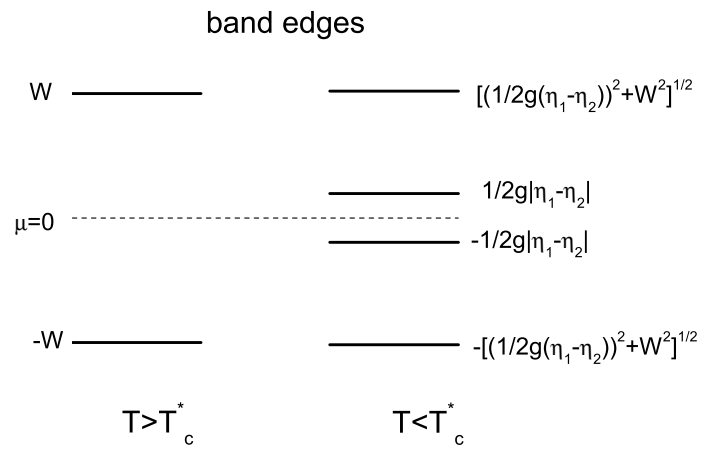

Fig. 10. The band edges for the cases $T>T_{c}^{*}$ (homogeneous phase) and $T<T_{c}^{*}$ (the double modulation case); $\mu=0, h=g$.

Contributions from both sublattices are present in the equation for the electron concentration in sublattices

$$
\begin{aligned}
n_{\alpha} & =\frac{1}{N / 2} \sum_{k \sigma}\left(\frac{1+\cos 2 \phi}{2}\left(e^{\beta \lambda_{k \alpha}-\mu}+1\right)^{-1}\right. \\
& \left.+\frac{1-\cos 2 \phi}{2}\left(e^{\beta \lambda_{k \beta}-\mu}+1\right)^{-1}\right)
\end{aligned}
$$

which is obtained using (24)-(27). Another equation which appears as a result of the averaging of the operator $S_{i \alpha}^{z}$ has the form

$$
\eta_{\alpha}=\frac{h-g n_{\alpha}}{2 \tilde{\lambda}_{\alpha}} \tanh \left(\frac{\beta \tilde{\lambda}_{\alpha}}{2}\right) ; \alpha \neq \beta
$$

The equations (28) and (29) form the system which plays role similar to the set of equations (7) for the uniform case. The grand canonical potential for the double modulation case can be written as follows:

$$
\begin{aligned}
\frac{2 \Phi}{N} & =-\frac{T}{N} \sum_{k} \ln \left(1+e^{-\frac{\lambda_{k 1}-\mu}{T}}\right)\left(1+e^{-\frac{\lambda_{k 2}-\mu}{T}}\right) \\
& -T \ln \left(4 \operatorname{ch} \frac{\beta \tilde{\lambda_{1}}}{2} \operatorname{ch} \frac{\beta \tilde{\lambda_{2}}}{2}\right)-g\left(n_{1} \eta_{1}+n_{2} \eta_{2}\right) .
\end{aligned}
$$

Solution of the obtained equation system (28) and (29) and investigation of the thermodynamically stable states was carried out numerically. It is established that phase transitions from the uniform to the low-temperature modulated phase can be of the second or the first order. It is illustrated in Fig. 11, where the phase transition lines at $\mu=0$ in the cases $\Omega=0$ and $\Omega \neq 0$ are shown (direct momentum summation is used for calculating the right hand sides of equations (28) and (30)).

The change of the phase transition order at the motion along the phase equilibrium curve is shown in Fig. 12, where the jump of the electron concentration $\delta n^{0}=$ $n_{1}^{0}-n_{2}^{0}$ in the transition point as a function of temperature is shown. The region where $\delta n^{0}=0$ corresponds to the second order phase transition (solid lines in Figs. 11 and 12), the region $\delta n^{0} \neq 0$ corresponds to the first order phase trasition (dashed lines in Figs. 11 and 12.)

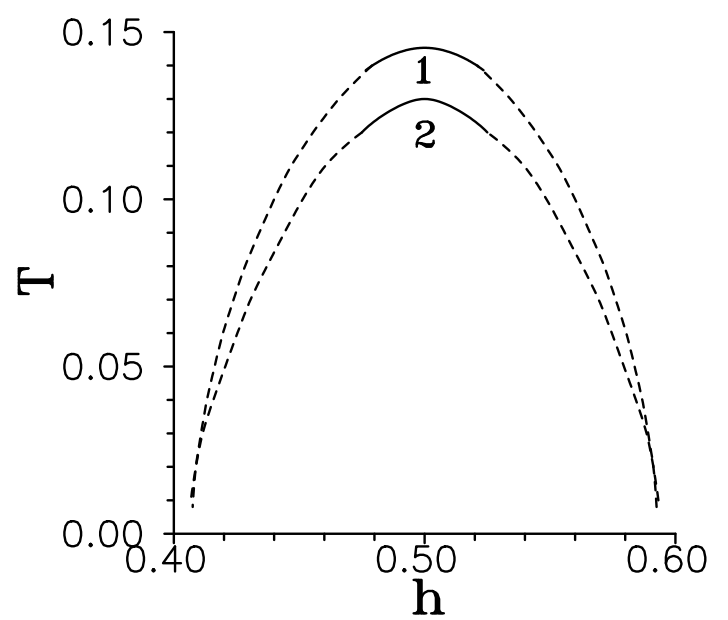

Fig. 11. The phase transition lines (solid and dashed lines are the lines of the second and the first order phase transitions, respectively) from the uniform phase to the phase with double modulation $(1-\Omega=0,2-\Omega=0.2)$.

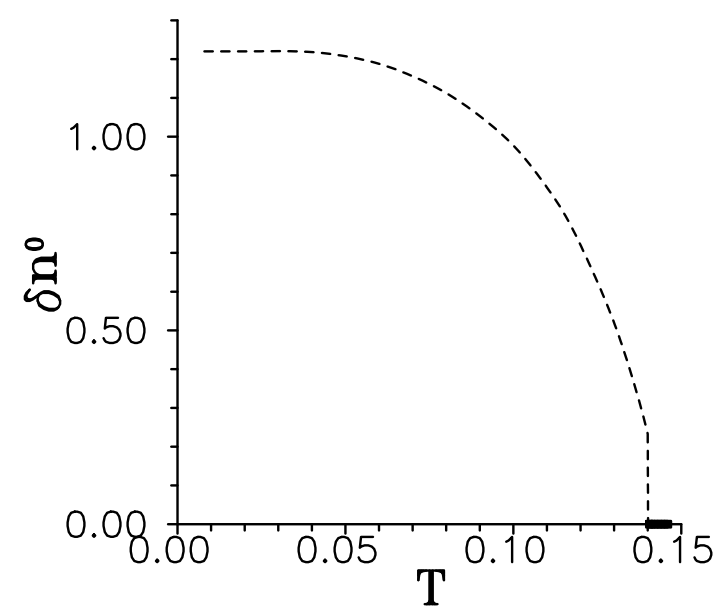

Fig. 12. The temperature dependence of the difference $\delta n^{0}=n_{1}^{0}-n_{2}^{0}$ along the phase transition line $(\Omega=0)$.

The difference $\delta n=n_{1}-n_{2}$ (as well as the difference $\delta \eta=\eta_{1}-\eta_{2}$ ) can play a role of the order parameter for modulated phase. Coming from equations (28) and (29), we can write the equations for $\delta n$ and $\delta \eta$ and separate after that the contributions of the first order:

$$
\delta n=-\frac{2}{N} \sum_{\sigma k}^{\prime} \frac{g}{2 t_{k}} \delta \eta\left[\left(e^{\beta\left(g \eta-t_{k}-\mu\right)}+1\right)^{-1}\right.
$$




$$
\begin{aligned}
& \left.-\left(e^{\beta\left(g \eta+t_{k}-\mu\right)}+1\right)^{-1}\right], \\
\delta \eta & =\frac{(h-g n)^{2}}{2 \lambda^{2}} g \delta n\left(\frac{1}{4}-\left\langle\sigma^{z}\right\rangle^{2}\right) \beta-g \delta n\left\langle\sigma^{z}\right\rangle \frac{\Omega^{2}}{\lambda^{3}}, \\
\left\langle\sigma^{z}\right\rangle & =\frac{1}{2} \tanh \frac{\beta \lambda}{2} ; \quad n=\frac{n_{1}+n_{2}}{2} ; \quad \eta=\frac{\eta_{1}+\eta_{2}}{2} .
\end{aligned}
$$

Here $\sum_{\sigma k}^{\prime}$ denotes the summation over the wave vector within the reduced Brillouin zone (when the condition $\left|k_{x}\right|+\left|k_{y}\right| \leq \frac{\pi}{a}, a$ is a lattice constant, is fulfilled). From the equations (31) and (32) we obtain the following condition of the appearance of nonzero solutions for $\delta n$ and $\delta \eta$ :

$$
\begin{aligned}
1 & =\frac{g}{N} \sum_{k \sigma} \frac{1}{t_{k}}\left(e^{\beta\left(g \eta-t_{k}-\mu\right)+1}\right)^{-1} \\
& \times\left[\beta g \frac{(h-g n)^{2}}{\lambda^{2}}\left(\frac{1}{4}-\left\langle\sigma^{z}\right\rangle^{2}\right)+g\left\langle\sigma^{z}\right\rangle \frac{\Omega^{2}}{\lambda^{3}}\right]
\end{aligned}
$$

(here the summation is carried out within the full Brillouin zone, which corresponds to the initial lattice).

Proceeding from equation (33), we can find a critical temperature $T_{c}^{*}$ as the maximum temperature (among the set of temperatures which are obtained for the different $h$ values) which fulfils this equation at the fixed value of the chemical potential. This temperature is the point of the second order phase transition to modulated phase at the corresponding value of the field $h$. Fig. 13 shows the dependences of the critical temperature $T_{c}^{*}$ on the chemical potential in the cases $\Omega=0$ and $\Omega \neq 0$. With the aim of comparison there are also shown in these figures the corresponding curves for the critical temperature $T_{c}$, which describes the transitions between the homogeneous phases (Fig. 6b). It is easy to see that $T_{c}^{*}>T_{c}$ for the $\mu$ values, which are less than the certain value $\left(|\mu|<\mu_{0} ; \mu_{0} \approx 0.4\right.$ in Fig. 13). Such condition is fulfilled for the electron band occupation, which is close to half-filling case. In this region the transition to modulated phase at the decrease of temperature occurs sooner than the described in paragraph II. A transition between the uniform phases; the last transition is realized when $|\mu|>\mu_{0}$.

Let us consider in more detail the situation in the symmetric case $\mu=0, h=g$, when in the high-temperature phase $n=1, \eta=0$. Equation (33) reduces now to the form

$$
1=-\frac{g^{2}}{\Omega} \tanh \frac{\beta \Omega}{2} \frac{1}{N} \sum_{k \sigma} \frac{1}{t_{k}} f\left(t_{k}\right)
$$

At the density of states $\rho(t)$ which is finite at $t=0$, in particular at the rectangular DOS $\rho(t)=\frac{1}{2 W}$, equation (34) in the low temperature region becomes

$$
1=\frac{g^{2}}{\Omega} \tanh \frac{\beta \Omega}{2} \frac{1}{2 W} \ln \frac{e W}{2 T},
$$
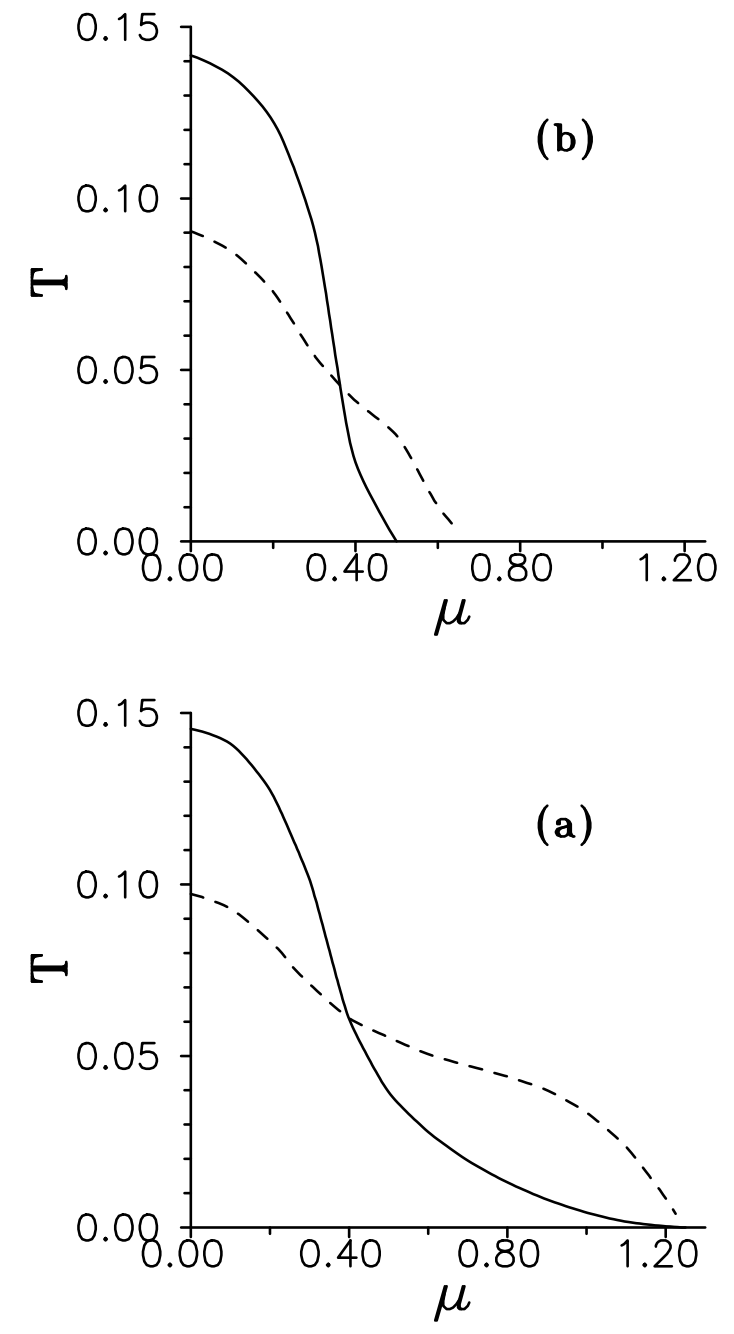

Fig. 13. The dependence of the critical temperature on the chemical potential; a) $\Omega=0$, b) $\Omega=0$.1. Solid line refers to the case of the phase with double modulation, dashed line refers to the transition into the homogeneous phase.

while at the presence of logarithmic singularity (16) we have the following equation:

$$
1=\frac{g^{2}}{\Omega} \tanh \frac{\beta \Omega}{2} \frac{2}{\pi^{2} W}\left(\ln \frac{2 W}{T}\right)^{2} .
$$

The critical temperatures $T_{c}^{*}$, obtained in these both cases are higher than the corresponding temperatures $T_{c}((15)$ and (20)) for the transitions between uniform phases. At high $\Omega$ values $T_{c}^{*}$ remains finite:

$$
T_{c}^{*} \approx \frac{W e}{2} \exp \left(-\frac{2 \Omega W}{g^{2}}\right)
$$

for the rectangular DOS (or $T_{c}^{*} \approx \frac{W}{2} \exp \left(-\frac{\Omega W \pi^{2}}{2 g^{2}}\right)$ in the case of the semi-elliptic DOS $\rho(t)=\frac{2}{W^{2} \pi^{2}} \sqrt{W^{2}-t^{2}}$ ) and 


$$
T_{c}^{*} \approx 2 W \exp \left(-\frac{\pi \sqrt{\Omega W}}{g \sqrt{2}}\right)
$$

in the case of the DOS with logarithmic singularity.

\section{CONCLUSIONS}

The pseudospin-electron model is investigated in the work in the weak coupling case $(g \ll W)$, when the electron band remains unsplit in the uniform state. The thermodynamically equilibrium states of the system are considered in the two regimes: the given chemical potential of electrons or the constant value of the electron concentration. In the $\mu=$ const regime the first order phase transitions between uniform phases (with the jumps of the electron concentration and pseudospin value) take place in the system when the chemical potential is placed within the electron band near its edges. In the case when $\mu$ is near the centre of the band, the phase with the doubling of the lattice period (the chess-board phase) can appear in the system at the lowering of temperature. The transition between the modulated and uniform phases can be of the first as well as of the second order depending on the magnitude of the field $h$ acting on pseudospins. For the $n=$ const regime the conditions of the appearance of the phase separation in the region of the phase transitions between uniform phases are established.

The obtained results, in general, are in accordance with the data of similar investigations for the FalicovKimball model at the small values of the coupling constant describing the interaction between the moving and localized particles (electrons). In $[18,19,21]$ the possibility of transitions into modulated or phase segregated phases was studied depending on the value of the mean electron concentration. It was shown that at small values of $n$ the phase separation can be realized, while near half-filling of the band the chess-board phase is preferable and, at last, at intermediate values of $n$ the appearance of phase with a noncommensurate modulation is possible. The transitions into this or that phase was fixed by the divergences of the corresponding susceptibilities (it should be mentioned in this connection that the usage of a such procedure did not give to the authors a possibility to reveal the thermodynamically stable states in the regions where the instabilities of either type (at $\mathbf{q}=0$ and $\mathbf{q}=(\pi / a, \pi / a, \ldots))$ are superimposed; this problem can be solved basing on the analysis of the behaviour of the grand canonical potential).

There exist, however, essential distinctions as compared with the results obtained for the FK model. Firstly, in papers $[17,18,20,21]$ the regime of the fixed concentration of localized particles was used (it corresponds in the case of the PEM to the fixation of the average value $\left.\left\langle S^{z}\right\rangle\right)$. Such a condition can lead to the phase separation by itself. Secondly, in contradiction to the FK model we take into account in the PEM the tunneling-like splitting. Besides the general decrease of the phase transition temperatures and narrowing of the segregation regions, its role manifests itself in the very conditions of the realization of phase transitions depending on the form of the density of electron states. In the cases when the electron DOS is finite at half-filling (does not possess a logarithmic singularity, that is characteristic of the $d=2$ space dimensionality) there exist such threshold values $\Omega_{c r}$ and $g_{\mathrm{cr}}$ that at $\Omega>\Omega_{\mathrm{cr}}$ or $g<g_{\mathrm{cr}}$ the phase transitions into segregated phases disappear.

Though the phase transitions in the PEM at weak coupling are similar to that revealed in this model in the case of the strong interaction, $g \gg W[14]$, the physical mechanisms of transitions are to a greater extent distinct. In the case of strong coupling the electron spectrum is always split due the to one-site interaction and the mechanism which ensures the advantage of the transition into the modulated phase or between the uniform ones is connected with the opposite in its character reconstruction of the electron spectrum in subbands and with the corresponding redistribution of the electron density of states between them. On the other hand, at the weak coupling a new phase which appears at the transition between uniform phases stabilizes due to the shift of the electron band as a whole, while the phase with a double modulation appears due to the energy gain at the splitting of the initial band at the Fermi level (the effect is similar to the Peierls instability at the interaction with phonons). Besides that, the dependences of the critical temperatures on the coupling constant $g$ are different in both cases: at $g \ll W T_{c}$ (or $T_{c}^{*}$ ) is proportional to $g^{2}$, while at $g \gg W$ the critical temperatures decrease $\left(\sim \frac{1}{g}\right)$ when $g$ increases (such a type of behaviour of $T_{c}^{*}$ for the FK model was obtained in [17]).

Let us mention that in the framework of the applied thermodynamocal approach we have performed the investigation of the phase transitions into uniform and chess-board phases only. Such transitions are connected with the instabilities described by divergencies of susceptibilities $\chi(0)=\frac{d n}{d \mu}$ and $\chi(\mathbf{Q})$ where $\mathbf{Q}=(\pi / a, \pi / a, \ldots)$. The consideration of orderings of another type (including the incommensurate ones) can be based on the investigation of singularities of the $\chi(\mathbf{q})$ function at any $\mathbf{q}$ value (the susceptibility $\chi(\mathbf{q})$ can be expressed in terms of the correlators $\left\langle T S_{i}^{z} S_{j}^{z}\right\rangle$ or $\left.\left\langle T n_{i} n_{j}\right\rangle\right)$. The corresponding investigation will be the subject of our subsequent consideration.
[1] K. A. Müller, Z. Phys. B 80, 193 (1990).

[2] J.E. Hirsch, S. Tang, Phys. Rev. B 40, 2179 (1989).

[3] M. Frick, W. van der Linden, L. Morgenstern, H. Raedt,
Z. Phys. B Cond. Matt. 81, 327 (1990).

[4] I. V. Stasyuk, A. M. Shvaika, Acta Phys. Pol. A 84, 293 (1993). 
[5] I. V. Stasyuk, A. M. Shvaika, E. Schachinger, Physica C 213, 57 (1993).

[6] I. V. Stasyuk, A. M. Shvaika, Physica C 235-240, 2173 (1994); Cond. Matt. Phys. (Lviv) iss. 3, 134 (1994).

[7] I. V. Stasyuk, A. M. Shvaika, O. D. Danyliv, Mol. Phys. Rep. 9, 61 (1995).

[8] I. V. Stasyuk, O. V. Velychko, Ukr. Fiz. Zh. 44, 772 (1999).

[9] I. V. Stasyuk, Yu. Havrylyuk, Cond. Matt. Phys. (Lviv) 2, 487 (1999).

[10] I. V. Stasyuk, A. M. Shvaika, Czech. J. Phys. 46, 961 (1996).

[11] V. M. Browning, Phys. Rev. B 56, 2860 (1997).

[12] O. Zahar, S. A. Kivelson, V. J. Emery, Phys. Rev. B 57, 1422 (1998).

[13] I. V. Stasyuk, A. M. Shvaika, J. Phys. Stud. 3, 177 (1999).

[14] I. V. Stasyuk, A. M. Shvaika, K. V. Tabunshchyk, Cond. Matt. Phys. (Lviv) 2, 109 (1999); Ukr. Fiz. Zh. 45, 520 (2000).
[15] Yu. A. Izyumov, B. M. Letfulov, J. Phys.: Cond. Matt. 2, 8905 (1990).

[16] L. M. Falicov, J. C. Kimball, Phys. Rev. Lett. 22, 997 (1969).

[17] U. Brandt, C. Mielsch, Z. Phys. B 75, 365 (1989); 79, 295 (1989); 82, 37 (1989).

[18] J. K. Freerics, Phys. Rev. B 47, 9263 (1993); 48, 14797 (1993).

[19] B. M. Letfulov, Eur. Phys. J. B 4, 447 (1998); 11, 423 (1999).

[20] J. K. Freericks, Ch. Gruber, N. Macris, Phys. Rev. B 60, 1617 (1999).

[21] J. K. Freericks, R. Lemanski, Phys. Rev. B 61, 13438 (2000).

[22] F. Gebhard, The Mott Metal-Insulator Transition: Models and Methods (Springer-Verlag, Berlin, 1997).

[23] S. N. Coppersmith, P.B. Littlewood, Phys. Rev. B 42, 3966 (1990).

[24] R. S. Markiewicz, Physica C 193, 323 (1992).

\title{
ФАЗОВІ ПЕРЕХОДИ У ПСЕВДОСПІН-ЕЛЕКТРОННІЙ МОДЕЛІ ПРИ СЛАБКІЙ ВЗАЄМОДІї
}

\author{
I. В. Стасюк, Т. С. Мисакович \\ Інститут фізики конденсованих систем Національной академї наук Украйни, \\ вул. Сбениіцького, 1, Львіб, 79011, Украйна \\ E-mail:ista@icmp.lviv.ua
}

\begin{abstract}
Досліджено термодинамічно рівноважні стани та фазові переходи у псевдоспін-електронній моделі з поперечним полем у наближенні слабкого зв'язку. У режимі постійного хемічного потенпіялу встановлено можливість, залежно від значення $\mu$, переходів першого роду між однорідними фазами та першого або, другого роду до фази з подвоєнням періоду кристалічної гратки. Проаналізовано умови виникнення в системі фазового розшарування при заданій середній електронній конџентрачії. Вивчено також вплив поперечного поля (яке пов'язане з розшепленням тунельного типу) на фазові переходи та умови їх реалізапіі. Побудовано фазові діяграми при різних густинах електронних станів у зоні провідности.
\end{abstract}

